Clinical Immunology, Poznan University of Medical Sciences, 27/33 Szpitalna St., 60-572 Poznan, Poland. E-mail: alszczep @amp.edu.pl

Support Statement: This study was supported by the Ministry of Science and Higher Education (Warsaw, Poland), grant number N402110534.

Statement of Interest: None declared.

\section{REFERENCES}

1 Undem BJ, Hunter DD, Liu M, et al. Allergen-induced sensory neuroplasticity in airways. Int Arch Allergy Immunol 1999; 118 150-153.

2 Virchow JC, Julius P, Lommatzsch $\mathrm{M}$, et al. Neurotrophins are increased in bronchoalveolar lavage fluid after segmental allergen provocation. Am J Respir Crit Care Med 1998; 158: 2002-2005.

3 Olgart Höglund C, Frossard N. Nerve growth factor and asthma. Pulm Pharmacol Ther 2002; 15: 51-60.

4 Noga O, Hanf G, Schaper C, et al. The influence of inhalative corticosteroids on circulating nerve growth factor, brain-derived neurotrophic factor and neurotrophin-3 in allergic asthmatics. Clin Exp Allergy 2001; 31: 1906-1912.

5 Lommatzsch M, Schloetcke K, Klotz J, et al. Brain-derived neurotrophic factor in platelets and airflow limitation in asthma. Am J Respir Crit Care Med 2005; 171: 115-120.

6 Muller GC, Pitrez PM, Teixeira AL, et al. Plasma brain-derived neurotrophic factor levels are associated with clinical severity in school age children with asthma. Clin Exp Allergy 2010; 40: 1755-1759.

7 Standardization of spirometry, 1994 update. American Thoracic Society. Am J Respir Crit Care Med 1995; 152: 1107-1136.

8 Szczepankiewicz A, Breborowicz A, Sobkowiak P, et al. Polymorphisms of two histamine-metabolizing enzymes genes and childhood allergic asthma: a case control study. Clin Mol Allergy 2010; 8: 14.

9 Joachim RA, Noga $\mathrm{O}$, Sagach $\mathrm{V}$, et al. Correlation between immune and neuronal parameters and stress perception in allergic asthmatics. Clin Exp Allergy 2008; 38: 283-290.

10 Koskela HO, Purokivi MK, Romppanen J. Neurotrophins in chronic cough: association with asthma but not with cough severity. Clin Respir J 2010; 4: 45-50.

\title{
Value of s-ACE, BAL lymphocytosis, and CD4+/CD8+ and CD103+CD4+/CD4+ T-cell ratios in diagnosis of sarcoidosis
}

\section{To the Editors:}

Sarcoidosis is one of the most common causes of idiopathic lung disease. The international standard for diagnosis of sarcoidosis is based on three criteria: 1) a compatible clinical and/or radiological presentation; 2) histological evidence of noncaseating granulomas; and 3) exclusion of other diseases that produce a similar histological or clinical picture [1]. The diagnosis of sarcoidosis is supported by an elevated CD4+/CD8+ T-cell ratio, bronchoalveolar lavage (BAL) lymphocytosis and elevated serum angiotensin-converting enzyme (s-ACE). However, these parameters are variable in sarcoidosis [2-4].

The integrin CD103 is expressed on CD4+ T-cells in BAL. Due to the high influx of circulating T-cells to the granulomata in sarcoidosis, a reduction in the fraction of BAL CD103+CD4+ T-cells has been suggested as a marker of sarcoidosis, in combination with lymphocytosis and CD4+/CD8+ ratio [5]. In selected groups of patients, decreases in the fraction of CD103+CD4+ T-cells are significantly associated with sarcoidosis [6, 7]. The purpose of this study was to evaluate these parameters as diagnostic markers of sarcoidosis in consecutive patients in a tertiary hospital setting.

The study included patients who underwent BAL with subsequent flow cytometric analyses at the Dept of Respiratory Medicine, Aarhus University Hospital (Aarhus, Denmark) from August 2007 until April 2009 ( $n=107)$. The descriptive parameters and paraclinical findings, including sACE $\left(\mathrm{U} \cdot \mathrm{L}^{-1}\right)$, BAL lymphocytosis (determined by differential count), and CD4+/CD8+ and CD103+CD4+/CD4+ T-cell ratios (determined using flow cytometry), were retrieved from case records. The analysis of CD4+/CD8+ ratio was not performed for five controls. The analysis of CD103+CD4+/CD4+ ratio was not performed for six controls. The lymphocyte count was not performed for 10 controls and two cases. The level of s-ACE was not measured for 34 controls. Two patients in the case group were treated with ACE inhibitors, and measurements of their s-ACE levels were not included in the analyses.

All patients who were included in the case group had biopsyconfirmed sarcoidosis with noncaseating granulomas that were found in transbronchial or mediastinal lymph node biopsies. 11 patients had sarcoidosis radiographic stage $0 / \mathrm{I}$ and eight patients had stage II/III. We did not include patients who were diagnosed with sarcoidosis on the basis of clinical features, radiology or BAL findings alone.

Patients in the control group were diagnosed with other pulmonary diseases $(n=88)$ : extrinsic allergic alveolitis $(n=12)$, idiopathic pulmonary fibrosis $(n=10)$, nonspecific interstitial pneumonitis $(n=6)$, desquamative interstitial pneumonitis $(n=2)$, lymphocytic interstitial pneumonitis $(n=1)$, collagen vascular disease with interstitial lung disease $(n=10)$, unclassified interstitial lung disease $(n=26)$, tuberculosis $(n=2)$, aspergillosis $(n=1)$ and other nongranulomatous lung diseases $(n=18)$. 
TABLE 1 Diagnostic values of single parameters

\begin{tabular}{|c|c|c|c|c|c|c|c|c|c|c|}
\hline & \multicolumn{2}{|r|}{ Cases } & \multicolumn{2}{|c|}{ Controls } & \multirow{2}{*}{$\begin{array}{l}\text { Optimal } \\
\text { cut-off }\end{array}$} & \multirow[t]{2}{*}{ Sensitivity \% } & \multirow[t]{2}{*}{ Specificity \% } & \multirow[t]{2}{*}{ PPV \% } & \multirow[t]{2}{*}{ NPV \% } & \multirow[t]{2}{*}{ p-value } \\
\hline & Subjects $n$ & Mean $(95 \% \mathrm{Cl})$ & Subjec & Mean $(95 \% \mathrm{Cl})$ & & & & & & \\
\hline Lymphocyte fraction \% & 17 & $28.1(18.9-37.4)$ & 73 & $15.7(12.1-19.3)$ & 13 & 71 & 68 & 34 & 91 & $<0.05$ \\
\hline CD4+/CD8+ & 19 & $6.1(5.2-7.0)$ & 83 & $3.7(2.9-4.5)$ & 3.8 & 68 & 73 & 37 & 91 & $<0.02$ \\
\hline CD103+CD4+/CD4+ & 19 & $0.23(0.20-0.27)$ & 82 & $0.32(0.28-0.36)$ & 0.22 & 63 & 76 & 29 & 88 & 0.064 \\
\hline S-ACE U. $\mathbf{L}^{-1}$ & 17 & $119.0(86.6-151.4)$ & 54 & $57.2(49.2-62.2)$ & 84 & 82 & 89 & 70 & 94 & $<0.001$ \\
\hline
\end{tabular}

PPV: positive predictive value; NPV: negative predictive value; s-ACE: serum angiotensin-converting enzyme.

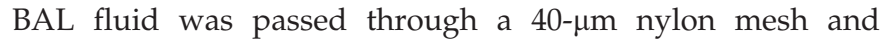
centrifuged. Subsequently, $100 \mu \mathrm{L}$ BAL fluid was stained with the following titrated antibodies: allophycocyanin-conjugated CD45 antibody (BD 55485; Becton Dickinson, Brondby, Denmark), AmCyan-conjugated CD3 antibody (BD 339186; Becton Dickinson), peridinin-chlorophyll protein complexconjugated CD4 antibody (BD 332772; Becton Dickinson), phycoerythrin-conjugated CD8 antibody (R0806; Dako, Glostrup, Denmark) and fluorescein isothiocyanate-conjugated CD103 antibody (BD 550259; Becton Dickinson). $1 \times 10^{6}$ events were analysed using a FACSCanto ${ }^{\mathrm{TM}}$ II system (Becton Dickinson). Lymphocytes, and CD3+, CD3+CD4+ and CD3+CD8+ T-cells were expressed as the fraction of CD45+ leukocytes. The number of CD3+CD4+ T-cells was divided by the number of CD3+CD8+ T-cells to calculate the CD4+/CD8+ ratio. The number of $\mathrm{CD} 3+\mathrm{CD} 4+\mathrm{CD} 103+$ cells was divided by the number of $\mathrm{CD} 3+\mathrm{CD} 4+\mathrm{T}$-cells to calculate the CD103+CD4+/CD4+ ratio.

The mean values of the diagnostic markers were compared using unpaired t-tests and $\mathrm{p}<0.05$ was considered statistically significant. For each parameter, the optimal cut-off point was defined as the point on a two-parameter receiver operating characteristic plot where sensitivity $=$ specificity. Sensitivity, specificity, positive predictive value and negative predictive value were subsequently calculated.

We detected significantly elevated lymphocyte percentages and CD4+/CD8+ ratios in BAL fluid and significantly elevated s-ACE for sarcoidosis patients compared with those in patients with other pulmonary diseases. However, we did not detect any significant differences in CD103+CD4+/CD4+ in BAL fluid between the patient groups (table 1). The combination of two or three diagnostic markers lowered the sensitivity (35-59\%) and increased the specificity (>90\%) (table 2). CD103+CD4+/ $\mathrm{CD} 4+$ did not correlate with radiographic staging.

KOLOPP-SARDA et al. [7] reported a sensitivity of $96 \%$ for the combination of CD103+CD4+/CD4+ $<0.31$ and CD4+/CD8+ $\geqslant 2.5$. In their study, all sarcoidosis patients $(n=18)$ had biopsyconfirmed diagnoses, $>10 \%$ lymphocytes and CD4+/CD8+ $\geqslant 2.5$. HERON et al. [6] reported a sensitivity of $66 \%$ and a specificity of $89 \%$ for the combined use of CD103+CD4+/CD4+ $<0.2$ with BAL CD4+/CD8+ $>3$ or BAL/peripheral blood CD4+/ $\mathrm{CD} 8+>2$ in 56 patients with biopsy-confirmed sarcoidosis.

Although BAL lymphocytosis is not a universal finding in sarcoidosis [2], the aforementioned studies included only patients with alveolar lymphocytosis. Among our sarcoidosis patients, five $(29 \%)$ out of 17 patients had a lymphocyte percentage $<10 \%$. Using our optimised cut-off levels of 0.22 and 3.8 for $\mathrm{CD} 103+\mathrm{CD} 4+/ \mathrm{CD} 4+$ and CD4+/CD8+, respectively, the sensitivity was $42 \%$ and the specificity was $91 \%$.

Addition of CD103+CD4+/CD4+ to the combination of s-ACE and CD4+/CD8+ decreased sensitivity (table 2).

When we applied the thresholds reported by HERON et al. [6] for BAL CD103+CD4+/CD4+ and CD4+/CD8+ to our data, we found a sensitivity of $39 \%$ (seven out of 18 patients) and specificity of $87 \%$ (68 out of 78 patients). Because BAL/ peripheral blood CD4+/CD8+ was not measured in our study, one patient with BAL CD4+/CD8+ $<3$ and CD103+CD4+/CD4+ $<0.20$ was excluded to allow comparative studies. One of the explanations for the lower sensitivity in our dataset may be the fact that patients were not pre-selected for lymphocytosis.

Using the criteria that have been proposed by KOLOPP-SARDA et al. [7], we found a sensitivity of $68 \%$ and a specificity of $79 \%$.

TABLE 2 Diagnostic values of selected combinations of parameters

\begin{tabular}{|c|c|c|c|c|c|c|}
\hline Combinations & Cases $\mathbf{n}$ & Controls $\mathrm{n}$ & Sensitivity \% & Specificity \% & PPV \% & NPV $\%$ \\
\hline CD103+CD4+/CD4+ $<0.22$ and lymphocyte fraction $>13 \%$ & 17 & 68 & 35 & 93 & 55 & 85 \\
\hline $\mathrm{CD} 103+\mathrm{CD} 4+/ \mathrm{CD} 4+<0.22$ and $\mathrm{CD} 4+/ \mathrm{CD} 8+>3.8$ & 19 & 78 & 42 & 91 & 53 & 87 \\
\hline $\mathrm{CD} 103+\mathrm{CD} 4+/ \mathrm{CD} 4+<0.22, \mathrm{CD} 4+/ \mathrm{CD} 8+>3.8$ and s-ACE $>84 \mathrm{U} \cdot \mathrm{L}^{-1}$ & 17 & 48 & 35 & 100 & 100 & 81 \\
\hline
\end{tabular}

PPV: positive predictive value; NPV: negative predictive value; s-ACE: serum angiotensin-converting enzyme. 
An earlier study demonstrated a significantly decreased level of CD103+CD4+/CD4+ in radiographic stage I sarcoidosis compared with healthy controls and significantly elevated levels in radiographic stage II/III sarcoidosis [5]. We found no significant differences in CD103+CD4+/CD4+ levels between the two radiographically staged groups in our study.

s-ACE is an important marker of sarcoidosis with high sensitivity but low specificity. A polymorphism in the ACE gene affects normal s-ACE concentration [8]. A homozygotic deletion in the promoter resulted in high levels of s-ACE (59.8 versus $32.2 \mathrm{U} \cdot \mathrm{L}^{-1}$ for the homozygous insertion allele; $\mathrm{p}<0.0001$ ) in a north German population [8]. Screening for this polymorphism is not common practice; however, the use of genotype-corrected normal ranges may increase the value of this parameter in the diagnosis and follow-up of sarcoidosis.

The combinations of CD103+CD4+/CD4+, CD4+/CD8+ and lymphocyte percentages that were tested in this study showed limited diagnostic value in this unselected group of sarcoidosis patients. Previously proposed criteria based on combinations of CD103+CD4+/CD4+ and CD4+/CD8+ had a lower sensitivity in this study than previously reported. s-ACE is an established marker of sarcoidosis and is the most useful of the markers that were investigated in this study.

Sarcoidosis is a disease with several phenotypes, which complicates the search for useful diagnostic markers. There remains an unmet need for new improved methods to support diagnosis when there is suspicion of sarcoidosis.

\section{Hyldgaard, S. Kaae, M. Riddervold, H.J. Hoffmann and O. Hilberg \\ Dept of Respiratory Medicine, Aarhus University Hospital,} Aarhus, Denmark.
Correspondence: C. Hyldgaard, Dept of Respiratory Medicine, Aarhus University Hospital, Norrebrogade 44, Aarhus 8000 C, Denmark. E-mail: charhyld@rm.dk

Statement of Interest: None declared.

\section{REFERENCES}

1 Statement on sarcoidosis. Joint Statement of the American Thoracic Society (ATS), the European Respiratory Society (ERS) and the World Association of Sarcoidosis and Other Granulomatous Disorders (WASOG) adopted by the ATS Board of Directors and by the ERS Executive Committee, February 1999. Am J Respir Crit Care Med 1999; 160: 736-755.

2 Kantrow SP, Meyer KC, Kidd P, et al. The CD4/CD8 ratio in BAL fluid is highly variable in sarcoidosis. Eur Respir J 1997; 10: 2716-2721.

3 Costabel U. CD4/CD8 ratios in bronchoalveolar lavage fluid: of value for diagnosing sarcoidosis? Eur Respir J 1997; 10: 2699-2700.

4 Welker L, Jorres RA, Costabel U, et al. Predictive value of BAL cell differentials in the diagnosis of interstitial lung diseases. Eur Respir J 2004; 24: 1000-1006.

5 Lohmeyer J, Friedrich J, Grimminger F, et al. Expression of mucosarelated integrin $\alpha^{\mathrm{E}} \beta_{7}$ on alveolar T cells in interstitial lung diseases. Clin Exp Immunol 1999; 116: 340-346.

6 Heron M, Slieker WA, Zanen P, et al. Evaluation of CD103 as a cellular marker for the diagnosis of pulmonary sarcoidosis. Clin Immunol 2008; 126: 338-344.

7 Kolopp-Sarda MN, Kohler C, De March AK, et al. Discriminative immunophenotype of bronchoalveolar lavage CD4 lymphocytes in sarcoidosis. Lab Invest 2000; 80: 1065-1069.

8 Ruprecht B, Schurmann M, Ziegenhagen MW, et al. Corrected normal values for serum ACE by genotyping the deletion-/ insertion-polymorphism of the ACE gene. Pneumologie 2001; 55: 326-332.

DOI: $10.1183 / 09031936.00144311$

\section{Surgical salvage following stereotactic body radiotherapy for early-stage NSCLC}

\section{To the Editors:}

For medically inoperable early-stage nonsmall cell lung cancer (NSCLC) patients, stereotactic body radiotherapy (SBRT) is the emerging treatment of choice. SBRT differs from conventional radiotherapy (RT) in that large doses of radiation are employed over a few (usually fewer than five, but sometimes up to 10) fractions to radioablate the tumour. Safe delivery of such profoundly damaging doses requires highly precise RT, in conjunction with image guidance and careful treatment planning.

Several prospective clinical trials have confirmed the feasibility, safety and efficacy of SBRT, consistently reporting local control rates of $80-95 \%$, comparable to surgical resection [1-3], although assessment of response is more complicated than it is following surgery [3]. Radiographic changes following SBRT differ from those following conventional RT, suggesting a unique histological response, and characterisation of these changes is a clinical challenge. Little is known of the histology of local recurrence following SBRT, since medically inoperable patients who fail locally cannot be salvaged surgically. Fineneedle aspiration biopsy provides only cytological information and is subject to false-negative results, while very frail patients may not even be able to tolerate the biopsy. Without histological confirmation, local failure (LF) is a clinical rather than a pathological diagnosis. In this report, we review our experience of four patients originally treated with SBRT who were salvaged surgically for their presumed LF.

From October 2004 to December 2010, we treated 209 patients with early (T1-2N0M0) NSCLC using SBRT on a prospective institution research ethics board-approved protocol. All patients were deemed medically inoperable by an experienced 